\title{
Análise da resistência a corrosão de revestimentos à base da liga de níquel depositados por soldagem em aço API 5L Gr B
}

\author{
Analysis of corrosion resistance of nickel alloy coatings deposited by welding \\ process on API 5L Gr B steel
}

Francisco de Assis Souza Neto ${ }^{1}$, Marcelo César Dias da Cunha' ${ }^{1}$, Aureliano Xavier dos Santos ${ }^{1}$, Theophilo Moura MacieL ${ }^{1}$, Marco Antonio dos Santos ${ }^{1}$, Renato Alexandre Costa de Santana ${ }^{2}$

\section{RESUMO}

Dentre os processos relacionados à manutenção dos equipamentos que operam nas indústrias do petróleo e gás, a soldagem é uma das mais importantes para recuperação de equipamentos de transporte, de armazenamento e de unidades de refino. Uma das aplicações deste processo neste setor é a aplicação de revestimento com ligas de níquel na superfície interna dos dutos para minimizar o efeito da corrosão. Objetivouse neste trabalho avaliar os efeitos dos diferentes parâmetros de soldagem sobre a resistência à corrosão de revestimentos a base de Níquel depositados sobre o aço API 5L Gr B, utilizado na fabricação dos dutos. Os depósitos de solda para obtenção dos revestimentos foram realizados pelo processo MIG variando os parâmetros de soldagem (Tensão de soldagem e Velocidade de alimentação do arame) com pré-aquecimento de $150^{\circ} \mathrm{C}$ e sem pré-aquecimento. Como ferramenta de otimização foi utilizado um Planejamento Fatorial $2^{2}$ e a Metodologia de Superfície de Resposta. Foi observado que os revestimentos obtidos sem pré-aquecimento mostraram Potencial de Corrosão mais positivo igual a $(-0,7 \mathrm{~V})$ e o mesmo comportamento de dissolução do revestimento quando comparado com os revestimentos obtidos com pré-aquecimento. As condições ótimas de soldagem obtidas com pré-aquecimentos foram com a menor Tensão de soldagem (22 V) e menor Velocidade alimentação de arame ( $5 \mathrm{~m} / \mathrm{min}$ ).

Palavras-chave: Soldagem MIG; Revestimento; Corrosão.

\begin{abstract}
Among the processes related to the maintenance of equipment operating in the oil and gas industries, welding is one of the most important for the recovery of transportation equipment, storage and refining units. One application of this process in the industry is to apply nickel plating alloy on the inner surface of the ducts to minimize the effects of corrosion. The aim of this study was to evaluate the effects of different welding parameters on the corrosion resistance coatings on nickel-based deposited on the steel API 5L Gr B, used in the manufacture of products. The weld deposits for obtaining coatings were carried out by varying the MIG welding parameters (welding voltage and wire feed speed) with preheat and $150^{\circ} \mathrm{C}$ without preheating. As optimization tool we used a Factorial Planning 22 and the Response Surface Methodology. It has been observed that the coatings obtained without preheating showed more positive corrosion potential equal to $(-0.7 \mathrm{~V})$, and the same dissolution behavior of the coating compared with coatings obtained using preheat. The optimum welding conditions obtained with pre-heating is to lower the welding voltage (22 V) and a lower wire feed speed ( $5 \mathrm{~m} / \mathrm{min})$.
\end{abstract}

Keywords: MIG Welding; Coating; Corrosion.

\footnotetext{
'Universidade Federal de Campina Grande - Unidade Acadêmica de Engenharia Mecânica - Campina Grande (PB) - Brasil ¿Universidade Federal de Campina Grande - Unidade Acadêmica de Biologia e Química - Cuité (PB) - Brasil

Autor correspondente: Aureliano Xavier dos Santos - Universidade Federal de Campina Grande - Unidade Acadêmica de Engenharia Mecânica - Av. Aprígio Veloso, 882 - Bairro Universitário - Campina Grande (PB) - CEP 58.429-140 - Brasil - E-mail: aurelliano@yahoo.com.br

Recebido: 22/04/2016 Aprovado: 11/01/2017
} 


\section{INTRODUÇÃO}

O petróleo, devido à sua composição química e à presença de diversas impurezas, apresenta um elevado grau de corrosividade. $\mathrm{O}$ processo de corrosão, principalmente por corrosão naftênica, que ocorre no interior dos dutos que transportam óleo ou gás, é um problema de relevância na indústria do petróleo e pode afetar de forma significativa a vida útil dos mesmos, causando aumento de custos de manutenção, produção e até a interrupção da tubulação para a substituição de alguma parte ${ }^{(1)}$.

Quando o fenômeno da corrosão não é controlado, ocorre o inevitável vazamento das tubulações, causando prejuízos econômicos e danos irreparáveis ao meio ambiente. Este aspecto exige dos diversos tipos de materiais empregados na fabricação dos dutos uma elevada resistência à corrosão ${ }^{(2)}$.

Uma das soluções empregadas para a correção dos equipamentos degradados é a recuperação através de materiais que apresentem elevada resistência à corrosão sendo estes depositados por soldagem. Dentre as técnicas de proteção de superfícies a do revestimento por soldagem é bem atrativa por oferecer a proteção através da aplicação de revestimentos unidos metalurgicamente ao substrato. Vários processos de soldagem vêm sendo utilizados para este fim tais como: Processo a laser, (LBW), processos GTAW ou TIG com alimentação automática de arame (frio e quente), processo GMAW (MIG/MAG) e o processo à Plasma por Arco Transferido (PTA). Todos eles têm a finalidade de aplicação de uma camada protetora de um material com propriedades superiores sobre o substrato ${ }^{(3-7)}$.

Em qualquer um desses casos, o desafio principal é encontrar materiais resistentes às severas condições de corrosão e ainda que atendam às diferentes condições de temperatura e de tensões ${ }^{(2)}$. Neste contexto, destaca-se a importância de um trabalho voltado para o estudo da compatibilidade metalúrgica de revestimentos a base de superligas de níquel, depositados por soldagem em chapas de aço C-Mn ${ }^{(8)}$. A partir de um estudo detalhado de vários processos de deposição, assim como do desempenho físico-metalúrgico destes revestimentos em segmentos de tubo será possível definir as melhores alternativas para suas aplicações como revestimento interno em tubulações ${ }^{(9)}$.

Objetivou-se nesse trabalho analisar o comportamento da resistência à corrosão de revestimentos a base da liga de níquel, Inconel 625, aplicados por soldagem MIG em aço API 5L Gr B com e sem preaquecimento do substrato avaliando o efeito da variação dos parâmetros de processo (Tensão e Velocidade de alimentação), através do uso de um planejamento experimental associado e a metodologia de superfície de resposta (MSR).

\section{MATERIAIS E MÉTODOS Materiais}

Utilizaram-se segmentos de um duto com $323 \mathrm{~mm}$ de diâmetro e $18 \mathrm{~mm}$ de espessura de aço API 5L Gr B. Para preparação dos corpos de prova o duto foi cortado nas dimensões $(100 \times 100 \times 18) \mathrm{mm}$ através do processo oxi-acetileno. A liga de níquel, Inconel 625, $\mathrm{ER} \mathrm{NiCrMo}{ }^{-3}$, na forma de arame maciço com diâmetro de $1,2 \mathrm{~mm}$ foi utilizada como metal de adição. $\mathrm{O}$ gás argônio foi usado como gás de proteção. As composições do metal de base e do metal de adição estão apresentadas nas Tabelas 1 e 2 , respectivamente.

\section{Procedimentos de Soldagem}

Os depósitos para obtenção dos revestimentos foram realizados pelo processo de soldagem MIG (Metal Inerte Gás). Utilizou-se uma fonte de soldagem modelo DIGI Plus A7 800 fabricada pela IMC Soldagem, contendo os seguintes acessórios: Fonte do tipo corrente contínua e tensão constante com capacidade de corrente máxima de $800 \mathrm{~A}$, sistema de aquisição de dados para o monitoramento dos parâmetros de soldagem e tartílope para deslocamento linear da tocha.

\section{Planejamento Experimental}

Para a otimização do processo de deposição da liga Inconel 625 , foi utilizado um planejamento fatorial completo $2^{2}$, em duplicata, sem ponto central, totalizando 8 experimentos para amostras com e sem pré-aquecimento sendo o pré-aquecimento de $150^{\circ} \mathrm{C}$, resultado em duas matrizes. Estes experimentos foram avaliados quantitativamente quanto à influência das variáveis de entrada: Tensão de Soldagem (U) e Velocidade de alimentação do arame $\left(\mathrm{V}_{\mathrm{a}}\right)$ sobre o Potencial de Corrosão (Ecorr) e Resistência à Polarização (Rp), bem como suas possíveis interações com a realização mínima de experimentos (10). Os experimentos foram realizados em ordem aleatória, para evitar o erro sistemático. A Tabela 3 apresenta as variáveis utilizadas no planejamento fatorial, níveis e suas codificações. Cada variável independente foi investigada para um nível baixo $(-1)$ e alto $(+1)$. Para a análise da regressão dos dados experimentais foi utilizado o software estatístico.

Tabela 1: Composição química (\% em massa) para aço ARBL API 5L GR B.

\begin{tabular}{|c|c|c|c|c|c|c|c|c|c|}
\hline \multirow{2}{*}{ API 5L GR B } & $\mathbf{C}$ & Si & Mn & P & S & Cr & Mo & Ni \\
\cline { 2 - 9 } & 0,20 & 0,23 & 0,8 & 0,015 & 0,018 & 0,064 & 0,008 & 0,07 \\
\hline
\end{tabular}

Tabela 2: Composição química (\% em massa) para Inconel 625 ( $\mathrm{ER} \mathrm{NiCrMo}^{-3}$ ).

\begin{tabular}{cccccccccccccc} 
& $\mathbf{C}$ & $\mathrm{Si}$ & $\mathrm{Mn}$ & $\mathbf{P}$ & $\mathbf{S}$ & $\mathrm{Cr}$ & $\mathrm{Mo}$ & $\mathrm{Ni}$ & $\mathrm{Al}$ & $\mathrm{Cu}$ & $\mathrm{Ti}$ & $\mathrm{Fe}$ & $\mathrm{Nb}+\mathrm{Ta}$ \\
\cline { 2 - 3 }
\end{tabular}


Tabela 3: Níveis e codificações das variáveis em estudo

\begin{tabular}{|c|c|c|}
\hline Variáveis & \multicolumn{2}{|c|}{ Níveis } \\
\hline Independentes & -1 & +1 \\
\hline Tensão $(\mathrm{V}) \mathrm{U}$ & 22 & 24 \\
\hline Vel. de alimentação $(\mathrm{m} / \mathrm{min}) \mathrm{Va}$ & 5 & 7 \\
\hline
\end{tabular}

Os parâmetros que foram mantidos fixos durante as deposições dos revestimentos foram: posição de soldagem (plana), ângulo de ataque da tocha de soldagem $\left(\theta=15^{\circ}\right.$ com a vertical), sentido de soldagem (empurrando), polaridade de corrente reversa (CC+), vazão do gás de proteção $(25 \mathrm{~L} / \mathrm{min})$ e distância bico de contato peça (DBCP) de $20 \mathrm{~mm}$.

O revestimento foi feito em três passes de solda depositados sem utilização de sobreposição de cada cordão depositado, conforme Fig. 1. O primeiro passe do revestimento foi depositado a uma distância de aproximadamente $4 \mathrm{~mm}$ da extremidade do corpo de prova. Para o depósito dos três cordões de revestimento nos corpos de prova sem pré-aquecimento esperou-se chegar até temperatura ambiente para realizar o próximo passe de revestimento, analogamente se fez para os corpos de prova com pré-aquecimento de $150^{\circ} \mathrm{C}$.

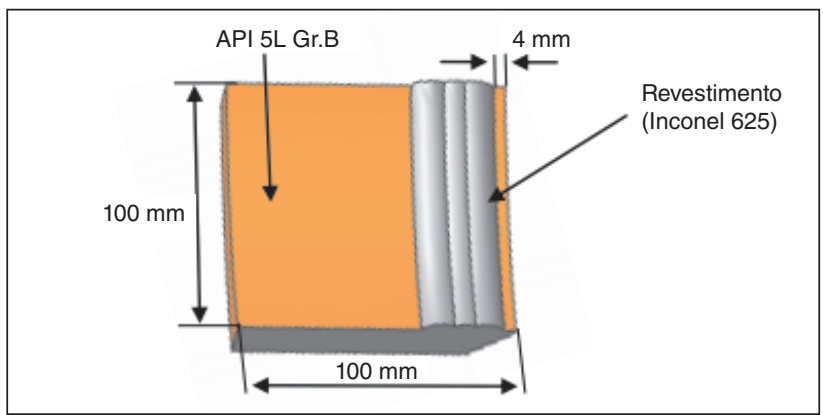

Figura 1: Revestimento MIG com três passes.

\section{Diluição}

As amostras obtidas, após corte transversal, foram avaliadas quanto à análise da Diluição. Para a medição do cálculo da diluição foi utilizado o software Image tool. A Fig. 2 mostra a

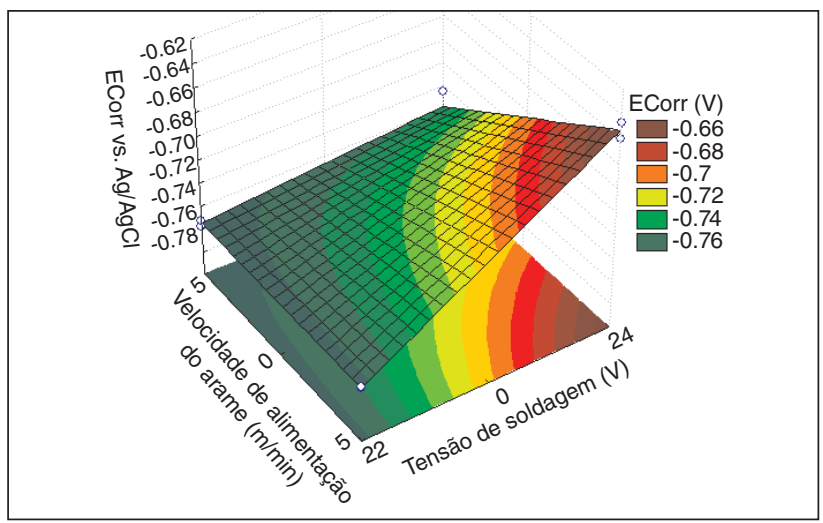

Figura 2: Representação esquemática da seção transversal do revestimento soldado ${ }^{(12)}$. representação esquemática da área do cordão de solda, indicando a largura $(\mathrm{L})$, reforço $(\mathrm{R})$, penetração $(\mathrm{P})$, área da zona fundida $\left(\mathrm{A}_{\mathrm{f}}\right)$, área do metal depositado $\left(\mathrm{A}_{\mathrm{d}}\right)$.

A diluição é o percentual do metal de base que se dissolve junto ao metal de adição formando a composição química do metal de solda e pode ser medida em função da área do metal de base fundida, conforme a Fig. 2 e a sua quantificação feita de acordo com a Eq. 1.

$$
\mathrm{D}(\%)=\left(\mathrm{A}_{\mathrm{f}} /\left(\mathrm{A}_{\mathrm{f}}+\mathrm{A}_{\mathrm{d}}\right)\right) \times 100
$$

\section{Composição química dos Revestimentos}

A análise da morfologia da superfície das camadas depositadas foi realizada pela técnica de microscopia eletrônica de varredura (MEV) usando um equipamento Philips XL-30. As composições químicas das ligas eletrodepositadas foram determinadas por energia dispersiva de raios $\mathrm{X}$ (EDX), usando o Programa Analytical QX-2000 acoplado ao MEV.

\section{Análise de Corrosão}

As análises de corrosão foram realizadas através das técnicas de Polarização Potenciodinâmica Linear (PPL) e Espectroscopia de Impedância Eletroquímica (EIE) em uma célula com três eletrodos através de uma medição eletroquímica utilizando o Equipamento Potenciostato/Galvanostato PG STATE30 da Autolab. O aço API 5L Gr.B revestido com a liga Inconel 625 foi usado como eletrodo de trabalho, um fio de platina como contra-eletrodo, e um eletrodo de prata cloreto de prata (Ag/ $\mathrm{AgCl}$ ) como eletrodo de referência. As curvas de Polarização Potenciodinâmica Linear a uma taxa de varredura de $1 \mathrm{mV} \mathrm{s}^{-1}$ e as medidas de Espectroscopia de Impedância Eletroquímica foram realizadas com os potenciais selecionados das curvas de PPL, com um intervalo de frequência de $10 \mathrm{kHz}$ a $0,004 \mathrm{~Hz}$ com uma amplitude de $0,01 \mathrm{~V}^{(11)}$. As análises de corrosão eletroquímica foram conduzidas no meio corrosivo contendo $60 \mathrm{ml}$ de solução $\mathrm{NaCl} 0,1 \mathrm{M}$ a temperatura ambiente. Para indicar a resistência à corrosão dos revestimentos obtidos pelo processo de soldagem, alguns parâmetros de caracterização relevantes foram analisados tais como: Resistência a polarização (Rp) e Potencial de corrosão (Ecorr).

\section{RESULTADOS E DISCUSSÃO Planejamento Experimental}

O delineamento experimental e Metodologia de Superfície de Resposta (MSR) foram utilizados para avaliar os efeitos da Tensão de Soldagem e Velocidade de Alimentação do arame sobre o Potencial de Corrosão (Ecorr) e Resistência a Polarização (Rp) dos revestimentos obtidos com pré-aquecimento e sem préaquecimento. Um modelo matemático de primeira ordem foi obtido pela análise estatística. Os coeficientes do modelo foram calculados através de regressão linear. Os resultados da resistência 
à polarização $\left(\mathrm{R}_{\mathrm{p}}\right)$ e Potencial de corrosão $\left(\mathrm{E}_{\text {corr }}\right)$ foram obtidos com auxílio da matriz de planejamento fatorial $2^{2}$ em duplicata usada para a otimização das variáveis Tensão $(\mathrm{U})$ e velocidade de alimentação (Va).

\section{Revestimentos obtidos sem pré-aquecimento}

A Tabela 4 apresenta a matriz de planejamento fatorial $2^{2} \mathrm{em}$ duplicata dos revestimentos obtidos sem pré-aquecimento e seus resultados experimentais com relação as variáveis dependentes: Diluição (D\%), \% de Ferro, \% Ni e Potencial de Corrosão (Ecorr) e Resistência a Corrosão (Rp).

\section{Efeitos das variáveis nos revestimentos sem pré-aquecimento.}

Os resultados do Potencial de Corrosão (Ecorr) e Resistência a Polarização (Rp) apresentados na Tabela 4 foram submetidos à análise de regressão não-linear para se obter os coeficientes para cada uma das variáveis independentes. Estimativas dos coeficientes com níveis mais alto que 95\% $(\mathrm{P}<0,05)$ foram incluídos no modelo final. O Potencial de Corrosão (Ecorr) e Resistência a Polarização (Rp) foram expressos na forma de uma função dos fatores independentes por um modelo matemático de $1^{\circ}$ ordem representado pelas Eqs. 2 e 3, onde (U) é Tensão de soldagem, (Va) Velocidade de alimentação do arame e (UVa) é a interação entre a Tensão de soldagem e Velocidade de alimentação do arame. Os valores em negrito nas equações correspondem aos valores estatisticamente significativos.

$$
\begin{aligned}
& \text { Ecorr }=-0,739+0,034 \cdot \mathrm{U}-0,026 \cdot \mathrm{Va}+0,026 \cdot \mathrm{U} \cdot \mathrm{Va} \\
& \mathrm{R}_{\mathrm{p}}=327887-63212 \cdot \mathrm{U}-63962 \cdot \mathrm{Va}-36662 \cdot \mathrm{U} \cdot \mathrm{Va}
\end{aligned}
$$

A análise de variância (ANOVA) para o modelo foi realizada com um nível de confiança de $95 \%$ para $\mathrm{P}<0,5$. O ajuste do modelo também foi expresso pelo coeficiente de regressão $\left(\mathrm{R}^{2}\right)$, que foi igual a $92 \%$ e $97 \%$ para o Potencial de Corrosão e Resistência à Polarização respectivamente. Os valores de $\mathrm{R}^{2}$ devem ser próximos da unidade (11). A análise de variância e de regressão dos dados demonstrou a significância estatística do modelo, justificando desta forma o uso do modelo de $1^{\text {a }}$ ordem para o estudo estatístico.

A Fig. 3 ilustra a superfície de resposta da Tensão de soldagem vs. Velocidade de alimentação tendo como resposta o Potencial de Corrosão. Foi observado que o valor ótimo encontrado foi obtido com a maior Tensão e Menor Velocidade de Alimentação.

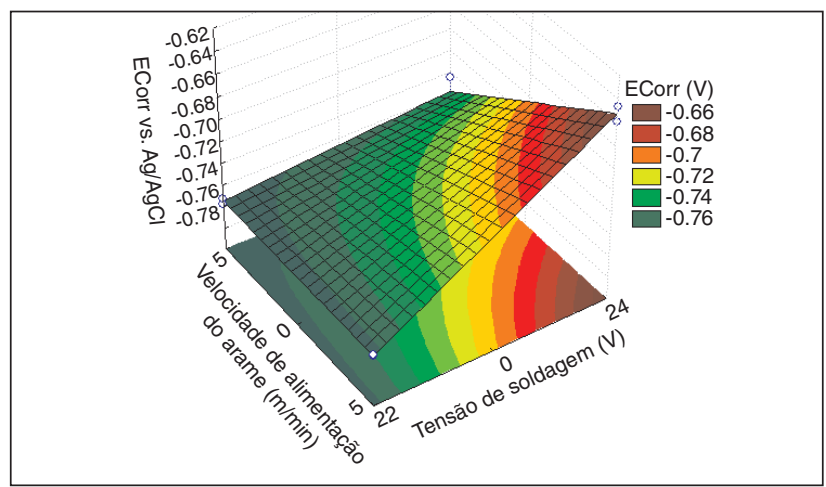

Figura 3: Superfície de resposta da Tensão de soldagem vs. Velocidade de alimentação tendo como resposta o potencial de corrosão.

A Fig. 4 ilustra a superfície de resposta da Tensão de soldagem $v$ s. Velocidade de alimentação do arame tendo como resposta a Resistência a Polarização. Com a diminuição da Tensão e diminuição da velocidade foram obtidos os melhores valores da Resistência a Polarização.

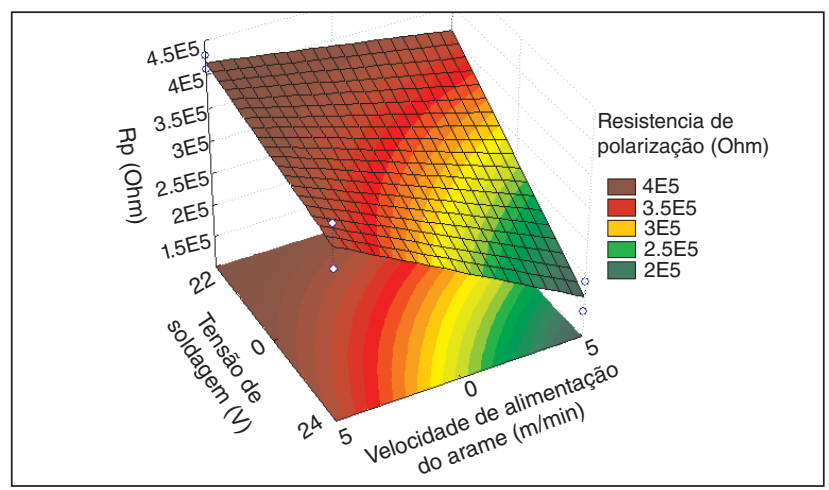

Figura 4: Superfície de resposta da Tensão de Soldagem vs. Velocidade de Alimentação tendo como resposta a Resistência de Polarização.

Tabela 4: Matriz do planejamento fatorial $2^{2}$ da liga de $\mathrm{Ni}$ sem pré-aquecimento $\left(25^{\circ} \mathrm{C}\right)$.

\begin{tabular}{|c|c|c|c|c|c|c|c|c|c|}
\multicolumn{4}{|c|}{ Variáveis Independentes } & \multicolumn{5}{|c|}{ Variáveis dependentes } \\
\hline Exp. & $\mathbf{U}(\mathbf{V})$ & $\mathbf{V a}(\mathbf{m} / \mathbf{m i n})$ & $\mathbf{R p}(\mathbf{K} \Omega)$ & Ecorr $(\mathbf{V})$ & $\mathbf{D} \%$ & $\%$ Fe & $\%$ Ni & $\%$ Cr \\
\hline 1 & 22 & 5 & 733,36 & $-0,773$ & 8,27 & 1,27 & 52,37 & 19,01 \\
\hline 2 & 24 & 5 & 596,60 & $-0,672$ & 6,00 & 1,17 & 38,48 & 13,83 \\
\hline 3 & 22 & 7 & 405,00 & $-0,784$ & 7,52 & 5,54 & 48,35 & 17,37 \\
\hline 4 & 24 & 7 & 403,20 & $-0,772$ & 7,85 & 6,46 & 49,69 & 17,87 \\
\hline 5 & 22 & 5 & 876,90 & $-0,774$ & 8,15 & 1,45 & 52,28 & 19,25 \\
\hline 6 & 24 & 5 & 611,02 & $-0,701$ & 7,34 & 1,35 & 37,12 & 13,03 \\
\hline 7 & 22 & 7 & 425,00 & $-0,777$ & 7,54 & 5,45 & 47,01 & 17,67 \\
\hline 8 & 24 & 7 & 370,70 & $-0,801$ & 7,94 & 6,39 & 48,14 \\
\hline
\end{tabular}




\section{Revestimentos obtidos com pré-aquecimento}

A Tabela 5 apresenta a matriz de planejamento fatorial $2^{2} \mathrm{em}$ duplicata dos revestimentos obtidos com pré-aquecimento e seus resultados experimentais com relação às variáveis dependentes: Diluição (D\%), \% de Ferro, \% Ni e Potencial de Corrosão (Ecorr) e Resistência a Corrosão (Rp).

\section{Efeitos das variáveis nos revestimentos com pré- aquecimento de $150^{\circ} \mathrm{C}$}

Os resultados do Potencial de Corrosão (Ecorr) e Resistência a Polarização (Rp) apresentados na Tabela 5 foram submetidos à análise de regressão não-linear para se obter os coeficientes para cada uma das variáveis independentes. Estimativas dos coeficientes com níveis mais alto que 95\% $(\mathrm{P}<0,05)$ foram incluídos no modelo final. O Potencial de Corrosão (Ecorr) e Resistência a Polarização (Rp) foram expressos na forma de uma função dos fatores independentes por um modelo matemático de $1^{\circ}$ ordem representado pelas Eqs. 4 e 5, onde (U) é Tensão de soldagem, (Va) Velocidade de alimentação do arame e (UVa) é a interação entre a Tensão de soldagem e Velocidade de alimentação do arame. Os valores em negrito nas equações correspondem aos valores estatisticamente significativos.

$$
\begin{aligned}
& \text { Ecorr }=\mathbf{- 0 , 7 4 8}+0,003 \cdot \mathrm{U}-0,014 \cdot \mathrm{Va}+\mathbf{0 , 0 3 2} \cdot \mathbf{U} \cdot \mathbf{V a} \\
& \mathrm{R}_{\mathrm{p}}=\mathbf{4 0 1 0 7 0}-\mathbf{2 3 6 4 7 0} \cdot \mathrm{U}+31320 \cdot \mathrm{Va}-27780 \cdot \mathrm{U} \cdot \mathrm{Va}
\end{aligned}
$$

A análise de variância (ANOVA) para o modelo foi realizada com um nível de confiança de $95 \%$ para $\mathrm{P}<0,5$. O ajuste do modelo também foi expresso pelo coeficiente de regressão $\left(\mathrm{R}^{2}\right)$, que foi igual a $84 \%$ e $95 \%$ para o Potencial de Corrosão e Resistência Polarização respectivamente. Os valores de $\mathrm{R}^{2}$ devem ser próximos da unidade ${ }^{(11)}$. A análise de variância e de regressão dos dados demonstrou a significância estatística do modelo, justificando desta forma o uso do modelo de $1^{\text {a }}$ ordem para o estudo estatístico.

A Fig. 5 ilustra a superfície de resposta da Tensão de soldagem vs. Velocidade de alimentação tendo como resposta o potencial de corrosão. Foi observado que o valor ótimo encontrado foi obtido com a menor tensão e menor velocidade.

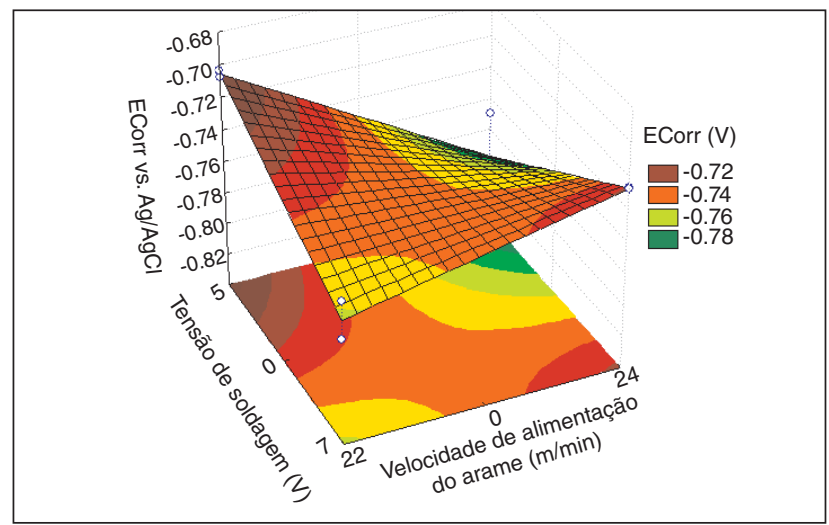

Figura 5: Superfície de resposta da Tensão de soldagem vs. Velocidade de alimentação tendo como resposta o Potencial de Corrosão.

A Fig. 6 ilustra a superfície de resposta da Tensão de soldagem vs. velocidade de alimentação do arame tendo como resposta a resistência de polarização. Com a diminuição da Tensão e da Velocidade foram obtidos os melhores resultados da Resistência a Polarização.

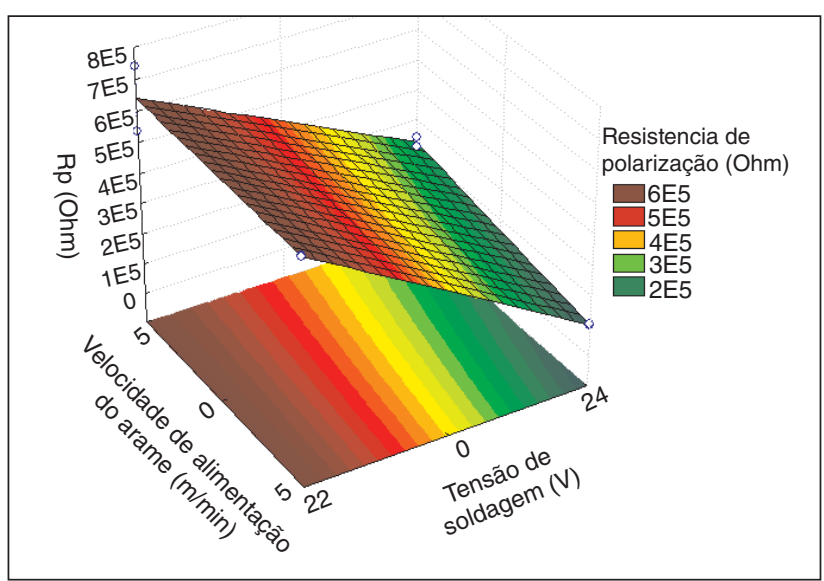

Figura 6: Superfície de resposta da Tensão de soldagem vs. Velocidade de alimentação tendo como resposta a resistência de polarização.

\begin{tabular}{|c|c|c|c|c|c|c|c|c|}
\hline \multicolumn{4}{|c|}{ Variáveis Independentes } & \multicolumn{5}{|c|}{ Variáveis Independentes } \\
\hline Exp. & $U(V)$ & $\mathrm{Va}(\mathrm{m} / \mathrm{min})$ & $R p(K \Omega)$ & Ecorr (V) & D \% & $\% \mathrm{Fe}$ & $\% \mathrm{Ni}$ & $\% \mathrm{Cr}$ \\
\hline 1 & 22 & 5 & 313,96 & $-0,770$ & 10,56 & 9,82 & 34,57 & 12,95 \\
\hline 2 & 24 & 5 & 423,19 & $-0,704$ & 7,53 & 3,5 & 50,16 & 18,09 \\
\hline 3 & 22 & 7 & 299,90 & $-0,807$ & 12,68 & 7,73 & 42,59 & 15,49 \\
\hline 4 & 24 & 7 & 351,04 & $-0,824$ & 10,11 & 4,56 & 52,15 & 18,76 \\
\hline 5 & 22 & 5 & 297,30 & $-0,707$ & 11,66 & 10,72 & 38,57 & 17,95 \\
\hline 6 & 24 & 5 & 328,85 & $-0,766$ & 7,07 & 4,5 & 49,14 & 16,18 \\
\hline 7 & 22 & 7 & 302,50 & $-0,824$ & 12,51 & 8,93 & 41,34 & 15,12 \\
\hline 8 & 24 & 7 & 393,00 & $-0,847$ & 9,17 & 5,47 & 50,01 & 18,54 \\
\hline
\end{tabular}

Tabela 5: Matriz do planejamento fatorial $2^{2}$ da liga de $\mathrm{Ni}$ com pré-aquecimento $\left(150^{\circ} \mathrm{C}\right)$. 


\section{Resistência à corrosão}

Os resultados da resistência à corrosão foram obtidos através das medições eletroquímicas obtidas através das técnicas de Polarização Potenciodinâmica Linear e Espectroscopia de Impedância Eletroquímica. A Fig. 7 apresenta as curvas de Polarização Potenciodinâmica Linear (PPL) das amostras otimizadas pelo planejamento experimental revestidas com Inconel 625 aplicadas com e sem pré-aquecimento, como também a curva de PPL do aço API 5L Gr.B sem revestimento. Observase que a amostra do Experimento 2 revestida com a liga Inconel 625 utilizando pré-aquecimento apresentou os piores resultados devido aos valores de potencial de corrosão menos positivos, ou seja, quanto mais positivo for o potencial de corrosão mais será retardado o início do processo de corrosão. Entretanto, a amostra do Experimento 2 com os revestimentos obtidos sem pré-aquecimento mostrou o mesmo comportamento de dissolução do revestimento que a amostra revestida com pré-aquecimento. O comportamento de dissolução é percebido pela comparação dos valores das correntes, representado no eixo das ordenadas da Fig. 7, referente aos dois revestimentos, onde corrente menor indica menor dissolução do material, logo a amostra sem pré-aquecimento mostrou melhor comportamento.

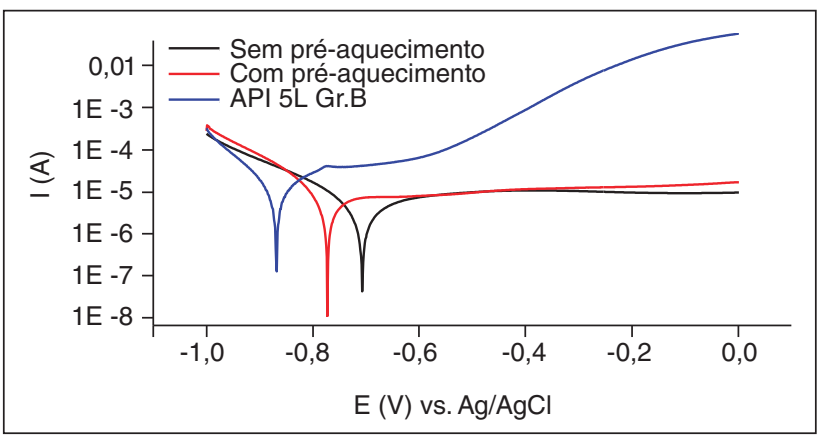

Figura 7: Curvas de Polarização das amostras dos revestimentos otimizadas obtidas com e sem pré-aquecimento, comparados com o substrato de aço API 5L Gr.B sem revestimento.

Observou-se através do diagrama de Nyquist, Fig. 8, que a amostra do Experimento 2 obtida sem pré-aquecimento apresentou o maior valor de Impedância, devido ao maior

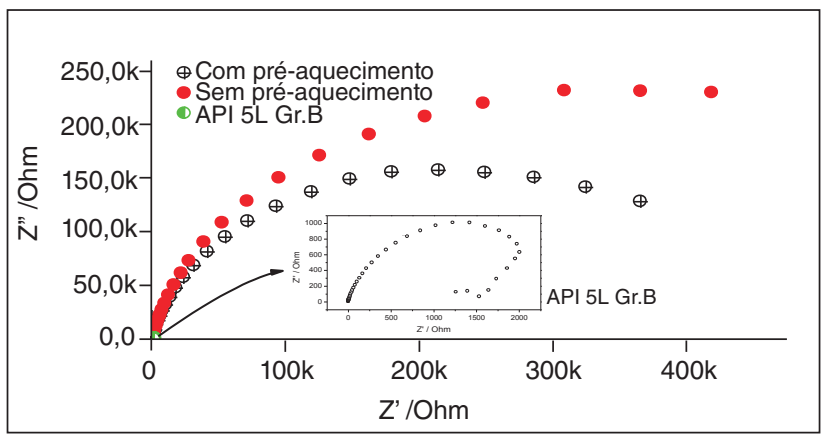

Figura 8: Diagrama de impedância relacionado aos revestimos otimizados obtidos com e sem preaquecimento, comparados com o substrato de aço carbono. semicírculo resistivo formado com o eixo, Z, o que indica que a amostra foi mais passiva quando comparadas às demais, devido à maior restrição da passagem de corrente na interface revestimento/solução, o que significa menor taxa de corrosão ou densidade de corrente de corrosão. Este fato está relacionado com o menor percentual de Ferro do revestimento, já que esta amostra apresentou uma menor diluição.

\section{CONCLUSÕES}

Com base nos resultados experimentais obtidos neste trabalho as conclusões são as seguintes:

O menor valor obtido para diluição foi de $6,0 \%$ na condição de $24 \mathrm{~V}$, velocidade do arame de $5 \mathrm{~m} / \mathrm{min}$, sem pré-aquecimento;

Com o pré-aquecimento de $150^{\circ} \mathrm{C}$ o valor ótimo encontrado tanto para o potencial de corrosão (Ecorr) quanto para a resistência a polarização (Rp), foi obtido com a menor Tensão de soldagem e menor velocidade de alimentação;

Sem aplicação de um pré-aquecimento o valor ótimo encontrado para o Potencial de Corrosão (Ecorr) foi igual a $0,7 \mathrm{~V}$ obtido com a maior Tensão e menor Velocidade de alimentação. E com a diminuição da Tensão da velocidade obtiveram-se os melhores valores de Resistência a Polarização(Rp);

Com a aplicação de um pré-aquecimento de $150^{\circ} \mathrm{C}$ obteve-se o melhor comportamento de resistência à corrosão para a maioria das amostras quando comparadas às sem pré-aquecimento, mantendo-se os outros dois parâmetros de soldagem iguais (Tensão de soldagem e Velocidade de alimentação do arame);

Entre os melhores resultados das duas matrizes (com e sem préaquecimento) percebe-se que a sem pré-aquecimento apresentou melhor comportamento perante a corrosão;

\section{AGRADECIMENTOS}

Agradecemos ao CNPq, CAPES pelo apoio financeiro.

\section{REFERENCES}

1. BERNARDES, R., Comportamento de Aços Inoxidáveis Comerciais e Inconel na Resistência à Corrosão Naftênica de Petróleos Nacionais: Uma Análise Fenomenológica. 2005. Dissertação (Mestre em Engenharia Metalúrgica de Materiais) - Universidade Federal do Rio de Janeiro, Rio de Janeiro.

1. GENTIL, V., Corrosão. Rio de Janeiro: Livros Técnicos e Científicos Editora S.A., p. 135 - 153, 2007.

2. ZIKIN, A., BADISCH, E., HUSSAINOVA, I., TOMASTIK, C., DANNINGER, H., Characterisation of TiC-NiMo reinforced Nibased hardfacing, Surf. Coatings Technol. 236 (2013) 36-44. doi:10.1016/j.surfcoat.2013.02.027

3. MENG, X., QIN, G., ZHANG, Y., FU, B., ZOU, Z., High speed TIG-MAG hybrid arc welding of mild steel plate, J. Mater. Process. Technol. 214 (2014) 2417-2424. doi:10.1016/j. jmatprotec.2014.05.020 
4. ELSAWY, A.H., Characterization of the GTAW fusion line phases for superferritic stainless steel weldments, J. Mater. Process. Technol. 118 (2001) 127-131. doi:10.1016/S09240136(01)00887-1.

5. SONG, J. L., LIN, S. B., YANG, C.L., MA, G.C., LIU,H., Spreading behavior and microstructure characteristics of dissimilar metals TIG welding-brazing of aluminum alloy to stainless steel, Mater. Sci. Eng. A. 509 (2009) 31-40. doi:10.1016/j.msea.2009.02.036.

6. BîRDEANU, A. V., CIUCĂ, C., PUICEA, A., Pulsed LASER(micro)TIG hybrid welding: Process characteristics, J. Mater. Process. Technol. 212 (2012) 890-902. doi:10.1016/j. jmatprotec.2011.11.014
7. CHAIRMAN, J. P. HUNT et al.; Nickel and Cobalt alloys. In: AWS (Usa) (Comp.). Welding Handbook. 3. ed. Miami: AWS, Cap. 4, 1996. (p. 217-288).

8. CASTI metals black book - North American Ferrous Data Edmonton: CASTI Pub. 2000. $4^{\text {th }}$ ed. Chapter 2 - Wrought carbon \& alloy steel metallurgy, (p. 50-51).

9. CALADO, V., MONTGOMERY, D.C., Planejamento de experimentos usando estatística. Rio de Janeiro: Editora e-papers, 2003.

10. ASTM Standards G5, 1994.

11. ANTUNES, R.A., ANTONIO, C., DE ALMEIDA, V., Influence of Testing Temperature on the Corrosion Behavior of Api $5 \mathrm{~L}$ X70 Pipeline, 4 (2013) 3252-3256. 